\title{
HYDRODYNAMICAL EQUATION FOR ELECTRON SWARMS
}

\author{
Joel L. Lebowitz \\ Department of Mathematics and Physics \\ Rutgers University \\ Piscataway, New Jersey 08854-8019 \\ e-mail: lebowitz@math.rutgers.edu \\ and \\ A. Rokhlenko \\ Department of Mathematics \\ Rutgers University \\ Piscataway, New Jersey 08854-8019 \\ e-mail: rokhlenk@math.rutgers.edu
}

\begin{abstract}
We study the long time behavior of light particles, e.g. an electron swarm in which Coulomb interactions are unimportant, subjected to an external field and elastic collisions with an inert neutral gas. The time evolution of the velocity and position distribution function is described by a linear Boltzmann equation (LBE). The small ratio of electron to neutral masses, $\epsilon$, makes the energy transfer between them very inefficient. We show that under suitable scalings the LBE reduces, in the limit $\epsilon \rightarrow 0$, to a formally exact equation for the speed (energy) and position distribution of the electrons which contains mixed spatial and speed derivatives. When the system is spatially homogeneous this equation reduces to and thus justifies, for $\epsilon$ small enough, the commonly used "two-term" approximation.
\end{abstract}




\section{Introduction}

The motion of an electron under the influence of an external electric field and elastic "collisions" with the "background" is a problem of both historic and contemporary interest. It arises in gas discharges in the laboratory and in the atmosphere, when the degree of ionization is so low that electron-electron and electron-ion interactions are negligible, the so called swarm approximation [Carron 1992, Allis 1956, Franklin 1976, Margenau and Hartman 1948]. Similar situations arise in the study of electron transport in solids in a semi-classical regime [Lorentz 1916, Sommerfeld 1956, Ben Abdolllah and Degon 1996, Ben Abdolllah et al 1996, Golse and Poupaud 1992]. In such situations it is often reasonable to treat the scatterers as if they were in thermal equilibrium. For electrons moving in a (almost) neutral gas with constant density $\rho$ this corresponds to the atoms having a Maxwellian velocity distribution $F(\mathbf{V} ; M)$ at a specified temperature $T$,

$$
F(\mathbf{V} ; M)=\left(\frac{2 \pi k T}{M}\right)^{-d / 2} \exp \left(-\frac{M V^{2}}{2 k T}\right)
$$

where $M$ is the mass of the scatterers, e.g. the neutral atoms in a noble gas, $k$ is the Boltzmann constant, and $d$ is the space dimension.

The time evolution of the electron distribution function is then given by a linear Boltzmann equation (LBE) of the form [Franklin 1976, Landau and Lifshits 1993],

$$
\frac{\partial f(\mathbf{r}, \mathbf{v}, t)}{\partial t}+\mathbf{v} \cdot \frac{\partial}{\partial \mathbf{r}} f-\frac{e \mathbf{E}}{m} \cdot \frac{\partial}{\partial \mathbf{v}} f=\int\left[K\left(\mathbf{v}, \mathbf{v}^{\prime}\right) f\left(\mathbf{r}, \mathbf{v}^{\prime}, t\right)-K\left(\mathbf{v}^{\prime}, \mathbf{v}\right) f(\mathbf{r}, \mathbf{v}, t)\right] d \mathbf{v}^{\prime}=\mathcal{K} f
$$

where $e, m$ are the charge and mass of an electron, $\mathbf{E}$ is the external field and $K$ gives the rate for scattering. The transition rate $K$ will depend on the nature of the scatterers but, for the case of elastic collisions, to which we restrict ourselves here, $K$ will satisfy detailed 
balance with respect to a Maxwellian velocity distribution for the electrons $F(\mathbf{v} ; m)$, at the same temperature as that of the neutrals, so that $K\left(\mathbf{v}, \mathbf{v}^{\prime}\right) F\left(\mathbf{v}^{\prime} ; m\right)=K\left(\mathbf{v}^{\prime}, \mathbf{v}\right) F(\mathbf{v} ; m)$. This ensures that for $E=0, F(\mathbf{v} ; m)$ is a stationary velocity distribution which will be approached by $f(\mathbf{v}, t)$ as $t \rightarrow \infty ; f(\mathbf{v}, t)$ is obtained from $f(\mathbf{r}, \mathbf{v}, t)$ by integrating over the spatial coordinate $\mathbf{r}$. We assume for simplicity that the electrons are confined to a large periodic box: $f$ is then a probability density with $\iint f(\mathbf{r}, \mathbf{v} ; t) d \mathbf{r} d \mathbf{v}=1$. It is also possible to consider other boundary conditions as well as electric and magnetic fields which are space and time dependent, but we shall not do so here. We shall also not consider here explicitly electrons in solids for which the scatterers are various kind of excitations [Ben Abdolllah and Degon 1996, Ben Abdolllah et al 1996, Golse and Poupaud 1992].

The central physical fact about the electron-neutral system is the great disparity in their masses: $\frac{m}{M}=\epsilon<10^{-4}$. Consequently the change in the speed of an electron in a typical collision, which is of order $\sqrt{\epsilon}$, will be very small, while the change in the velocity direction will be large, becoming independent of $\epsilon$ when $\epsilon \rightarrow 0$ [Franklin 1976, Landau and Lifshits 1993]. This suggests approximating the integral operator in (2) by expanding the right side of (2) in powers of $\epsilon$ and dropping "small terms" in $\epsilon$. There is a certain amount of ambiguity in carrying out such an expansion, arising from the uncertainty of how to treat the dependence of the unknown $f$ on $\epsilon$. A reasonable choice gives

$$
\frac{\partial f}{\partial t}+\mathbf{v} \cdot \frac{\partial f}{\partial \mathbf{r}}-\frac{e \mathbf{E}}{m} \cdot \frac{\partial f}{\partial \mathbf{v}}=\frac{\epsilon \rho}{v^{2}} \frac{\partial}{\partial v}\left[v^{4} \sigma(v)\left(f_{0}+\frac{k T}{m v} \frac{\partial f_{0}}{\partial v}\right)\right]+v \rho \sigma(v)\left(f_{0}-f\right) .
$$

In (3) $v=|\mathbf{v}|, f_{0}(\mathbf{r}, v, t)$ is the average of $f(\mathbf{r}, \mathbf{v}, t)$ over angles and $\sigma(v)$ is the collision cross section, see [Margenau and Hartman 1948, Ginzburg and Gurevich 1960]. The dependence of $f$ on $\epsilon$ is then to be determined from the solution of (3). 
There is, however, very little control over this expansion. In fact eq.(3) is not necessarily positivity preserving. Nevertheless eq.(3) yields reasonable answers for the stationary velocity distribution of the electrons, $\bar{f}(\mathbf{v})$. The latter can be obtained explicitly in the so-called "two-term" approximation [Lorentz 1916, Margenau and Hartman 1948, Allis 1956, Carron 1991], in which one keeps only the first two terms in a spherical harmonic expansion of $\bar{f}(\mathbf{v})$. This distribution was first found by Druyvesteyn [Druyvesteyn 1930, Druyvesteyn and Penning 1940], who considered the case $T=0, \sigma=$ const, and was later generalized by Davydov [Davydov 1935] for all $T$ and $\sigma$. For an analysis of the error made by the two term approximation to (3) see [Rokhlenko and Lebowitz 1997, Rokhlenko submitted].

A somewhat different approach to this problem was taken by Koura [Koura 1987]. Starting with a two term $\epsilon$-expanded kinetic equation (slightly different than what is obtained from (3)) he observed, that after a scaling of space, time and electric field and neglecting the time variation of the first harmonic, one is led to an equation in which $\epsilon$ does not appear at all. Koura then argued that the actual physical quantities of interest in an electron swarm should have a similar simple scaling dependence on $\epsilon$, when $\epsilon$ is small. This has the advantage of permitting more efficient computer simulations at larger than realistic value of $\epsilon$. Doing simulations for several values of $\epsilon$ Koura found good agreement with results from the scaled two term approximation for $\epsilon \lesssim 10^{-2}$.

In this note we shall use the same scaling as Koura but apply it directly to (2) without any other approximation. This is in the same spirit as the scalings used for electrons in solids [Ben Abdolllah and Degon 1996, Ben Abdolllah et al 1996, Golse and Poupaud 
1992]. It is based on a formulation of space-time scalings now commonly used to obtain a "reduced hydrodynamic description" from a more detailed microscopic one, see [Spohn 1995].

\section{One-dimensional problem}

To simplify matters we shall first consider the one dimensional version of (2), corresponds to hard collisions of point particles with masses $m$ and $M=m / \epsilon$,

$$
\begin{gathered}
\frac{\partial f(x, \mathbf{v}, t)}{\partial t}+\mathbf{v} \frac{\partial f(x, \mathbf{v}, t)}{\partial x}-\frac{e E}{m} \frac{\partial f(x, \mathbf{v}, t)}{\partial \mathbf{v}}= \\
\rho \int_{-\infty}^{\infty}|\mathbf{v}-V|\left\{f\left[x, \frac{2 V-(1-\epsilon) \mathbf{v}}{1+\epsilon}, t\right] F\left[\frac{(1-\epsilon) V+2 \epsilon \mathbf{v}}{1+\epsilon} ; \frac{m}{\epsilon}\right]-f(x, \mathbf{v}, t) F(V ; m / \epsilon)\right\} d V
\end{gathered}
$$

where $\mathbf{v} \in \mathbb{R}$. Eq. (5) is to be solved subject to some initial condition $f(x, \mathbf{v}, 0)$.

To obtain the behavior of $f$ for long times we rescale our variables by setting $y=\sqrt{\epsilon} x$, $\tau=\epsilon t$ (diffusive scaling [Spohn 1995]) and $E=\sqrt{\epsilon} E^{*}$ (the field has to be small on this

scale for the energy to remain bounded when the time is of order $\epsilon^{-1}$ ). We now define the even and odd parts of the velocity distribution function

$$
\begin{gathered}
\phi_{\epsilon}(y, v, \tau)=\frac{1}{2 \sqrt{\epsilon}}[f(y / \sqrt{\epsilon}, v, \tau / \epsilon)+f(y / \sqrt{\epsilon},-v, \tau / \epsilon)], \\
\sqrt{\epsilon} \psi_{\epsilon}(y, v, \tau)=\frac{1}{2 \sqrt{\epsilon}}[f(y / \sqrt{\epsilon}, v, t / \epsilon)-f(y / \sqrt{\epsilon},-v, t / \epsilon)],
\end{gathered}
$$

where $v=|\mathbf{v}|$ and we have put $f \rightarrow \frac{1}{\sqrt{\epsilon}} f$ to preserve the normalization: in the scaled variable $y$ the system is in a periodic box of size $L$ independent of $\epsilon$. By changing the integration variable in (3) we then obtain two coupled equations for $\phi_{\epsilon}$ and $\psi_{\epsilon}$,

$$
\frac{\partial \phi_{\epsilon}(y, v, \tau)}{\partial \tau}+v \frac{\partial \psi_{\epsilon}}{\partial y}-\frac{e E^{*}}{m} \frac{\partial \psi_{\epsilon}}{\partial v}=
$$




$$
\begin{gathered}
\epsilon^{-1} \rho \sqrt{\frac{m}{2 \pi k T}} \int_{-\infty}^{\infty}|v-V|\left\{\left(\frac{1+\epsilon}{1-\epsilon}\right)^{2} \phi_{\epsilon}\left[y, \frac{(1+\epsilon) v-2 V}{1-\epsilon}, \tau\right]-\phi_{\epsilon}(y, v, \tau)\right\} e^{-\frac{m V^{2}}{2 \epsilon k T}} d V, \\
\epsilon \frac{\partial \psi_{\epsilon}(y, v, \tau)}{\partial \tau}+v \frac{\partial \psi_{\epsilon}}{\partial y}-\frac{e E^{*}}{m} \frac{\partial \phi_{\epsilon}}{\partial v}= \\
-\rho \sqrt{\frac{m}{2 \pi k T}} \int_{-\infty}^{\infty}|v-V|\left\{\left(\frac{1+\epsilon}{1-\epsilon}\right)^{2} \psi_{\epsilon}\left[y, \frac{(1+\epsilon) v-2 V}{1-\epsilon}, \tau\right]+\psi_{\epsilon}(y, v, \tau)\right\} e^{-\frac{m V^{2}}{2 \epsilon k T}} d V .
\end{gathered}
$$

We assume now that the initial distribution is such that $\phi_{\epsilon}$ and $\psi_{\epsilon}$ have well defined limits $\phi(y, v, 0)$ and $\psi(y, v, 0)$ as $\epsilon \rightarrow 0$. Taking now formally the limit $\epsilon \rightarrow 0$ on both sides of (7), keeping $\tau, y$ and $E^{*}$ fixed we get the limiting equations,

$$
\begin{gathered}
\frac{\partial \phi(y, v, \tau)}{\partial \tau}+v \frac{\partial \psi}{\partial y}-\frac{e E^{*}}{m} \frac{\partial \psi}{\partial v}=2 \rho \frac{\partial}{\partial v}\left[v^{2}\left(\phi+\frac{k T}{m} \frac{\partial \phi}{\partial v}\right)\right] \\
v \frac{\partial \phi}{\partial y}-\frac{e E^{*}}{m} \frac{\partial \phi}{\partial v}=-2 \rho v \psi(y, v, \tau)
\end{gathered}
$$

valid for $v \geq 0$. Solving (8b) for $\psi$ and substituting into (8a) we get the reduced equation for the even part of the distribution $\phi(y, v, \tau)$, in terms of the scaled space, time and electric field

$$
\frac{\partial \phi}{\partial \tau}+\frac{1}{\rho} \frac{\partial}{\partial y}\left(\frac{e E^{*}}{m} \frac{\partial \phi}{\partial v}-\frac{v}{2} \frac{\partial \phi}{\partial y}\right)=\frac{\partial}{\partial v}\left\{\left[\left(\frac{e E^{*}}{m}\right)^{2} \frac{1}{2 \rho v}+\frac{k T}{m} 2 \rho v\right] \frac{\partial \phi}{\partial v}+2 \rho v^{2} \phi\right\}
$$

with the boundary condition at $v=0$,

$$
\frac{\partial \phi(y, 0, \tau)}{\partial y}=\frac{e E^{*}}{m} \lim _{v \rightarrow+0} \frac{1}{v} \frac{\partial \phi(y, v, \tau)}{\partial v}
$$

and the normalization

$$
\int_{0}^{L} d y \int_{0}^{\infty} \phi(y, v, \tau) d v=1 / 2
$$

The condition (10) at $v=0$ follows from the (assumed) continuity of the distribution $f$ at $\mathbf{v}=0$. 
Let us introduce the rescaled electron density $n(y, \tau)$, drift $u(y, \tau)$, and mean speed $w(y, \tau):$

$$
\begin{gathered}
n(y, \tau)=2 \int_{0}^{\infty} \phi(y, v, \tau) d v \\
n(y, \tau) u(y, \tau)=2 \int_{0}^{\infty} v \psi(y, v, \tau) d v, \quad n(y, \tau) w(y, \tau)=2 \int_{0}^{\infty} v \phi(y, v, \tau) d v .
\end{gathered}
$$

Integrating Eq. (9) over velocities, we obtain the continuity equations

$$
\frac{\partial n(y, \tau)}{\partial \tau}+\frac{\partial(n u)}{\partial y}=0
$$

To obtain $u$ we substitute (8b) into (11) and integrate over $v$,

$$
u=-\frac{e \phi(y, 0, \tau)}{m \rho n(y, \tau)} E^{*}-\frac{w(y, \tau)}{2 \rho n(y, \tau)} \frac{\partial n}{\partial y}-\frac{1}{2 \rho} \frac{\partial w(y, \tau)}{\partial y} .
$$

One can identify in (13) the factors in front of $E^{*}, \frac{\partial n}{\partial y}, \frac{\partial w}{\partial y}$ as respectively the mobility, diffusion coefficient, and a parameter related to thermodiffusivity [Golant et al. 1980].

The right side of eq.(9) coresponds to a drift-diffusion in "speed space" with a diffusion coefficient given by the term in the square brackets and with a drift $-2 \rho v^{2}$. The spatially hompgeneous stationary solution of (9) has the form

$$
\bar{\phi}(v)=C \exp \left[-\int_{0}^{v} \frac{s^{3} d s}{\frac{k T}{m} s^{2}+\left(\frac{e E^{*}}{2 m \rho}\right)^{2}}\right],
$$

similar to the Davydov distribution for $d=3$, c.f. eq. (21). Note that for $E^{*}=0$, i.e. no external field, $\bar{\phi}(v)=F(v ; m)$ as it should.

\section{Three dimensions}

In $d=3$ the right side of the LBE takes the form

$$
\mathcal{K} f=\rho \iint|\mathbf{v}-\mathbf{V}| \sigma(\mathbf{v}-\mathbf{V})\left[f\left(\mathbf{v}^{\prime}, t\right) F\left(\mathbf{V}^{\prime} ; M\right)-f(\mathbf{v}, t) F(\mathbf{V} ; M)\right] d \mathbf{V} d \hat{\omega}
$$


The angular integration is over the scattering solid angle $\hat{\omega}$ of the electron in the center of mass coordinate system, $\sigma(\mathbf{v})$ is the electron-neutral differential collision cross section, and we have

$$
\begin{aligned}
\mathbf{v}^{\prime} & =\mathbf{v}+\frac{2}{1+\epsilon} \hat{\omega}[\hat{\omega} \cdot(\mathbf{V}-\mathbf{v})], \\
\mathbf{V}^{\prime} & =\mathbf{V}-\frac{2 \epsilon}{1+\epsilon} \hat{\omega}[\hat{\omega} \cdot(\mathbf{V}-\mathbf{v})] .
\end{aligned}
$$

Carrying out a similar analysis as for $d=1$ we separate $f$ into a spherically symmetric part $\phi_{\epsilon}(\mathbf{y}, v, \tau)$ and a remainder $\psi_{\epsilon}(\mathbf{y}, \mathbf{v}, \tau)$, i.e. we set

$$
\begin{aligned}
f(\mathbf{y} / \sqrt{\epsilon}, \mathbf{v}, t / \epsilon) & =\epsilon^{3 / 2}\left[\phi_{\epsilon}(\mathbf{y}, v, \tau)+\sqrt{\epsilon} \psi_{\epsilon}(\mathbf{y}, \mathbf{v}, \tau)\right], \\
\phi_{\epsilon}(\mathbf{y}, v, \tau) & =\frac{\epsilon^{-3 / 2}}{4 \pi} \int f(\mathbf{y} / \sqrt{\epsilon}, \mathbf{v}, t / \epsilon) d \Omega .
\end{aligned}
$$

In (16) the integration is over the unit sphere specifying the orientation of the vector $\frac{\mathbf{v}}{v}$. Taking now formally the limit $\epsilon \rightarrow 0$ we obtain, in terms of the rescaled variables, a set of equations for $\phi$ and $\psi$ entirely analogous to (8),(9),

$$
\begin{gathered}
\frac{\partial \phi}{\partial \tau}-\frac{v}{3 \rho \sigma} \nabla_{y}^{2} \phi+\frac{e}{3 m \rho} \mathbf{E}^{*} \cdot \nabla_{y}\left[\frac{1}{\sigma} \frac{\partial \phi}{\partial v}+\frac{1}{v^{2}} \frac{\partial}{\partial v}\left(\frac{v^{2} \phi}{\sigma}\right)\right]= \\
\frac{1}{v^{2}} \frac{\partial}{\partial v}\left[\left(\frac{e E^{*}}{m}\right)^{2} \frac{v}{3 \sigma \rho} \frac{\partial \phi}{\partial v}+\rho v^{4} \sigma(v)\left(\phi+\frac{k T}{m v} \frac{\partial \phi}{\partial v}\right)\right], \\
\psi=\frac{1}{\rho \sigma(v) v}\left(\frac{e}{m} \mathbf{E}^{*} \cdot \nabla_{\mathbf{v}} \phi-\mathbf{v} \cdot \nabla_{\mathbf{y}} \phi\right) .
\end{gathered}
$$

We assume the collisions to be spherically symmetric, so $\sigma(v)=4 \pi \sigma(\mathbf{v})$ is the total cross section.

The spatial density and current are given by

$$
n(\mathbf{y}, \tau)=4 \pi \int_{0}^{\infty} v^{2} \phi(\mathbf{y}, v, \tau) d v, \quad n(\mathbf{y}, \tau) \mathbf{u}(\mathbf{y}, \tau) .
$$


They satisfy equation of continuity

$$
\frac{\partial n(\mathbf{y}, \tau)}{\partial \tau}+\nabla_{y}[n \mathbf{u}]=0
$$

The electron drift $\mathbf{u}$ has the form

$$
\begin{gathered}
\mathbf{u}=\mu \mathbf{E}^{*}-D \frac{1}{n} \nabla_{y} n-\frac{1}{3 \rho} \nabla_{y}\left\langle\frac{v}{\sigma(v)}\right\rangle, \\
\mu=\frac{e}{3 \rho m}\left\langle\frac{1}{v^{2}} \frac{d}{d v}\left[\frac{v^{2}}{\sigma(v)}\right]\right\rangle, \quad D=\frac{1}{3 \rho}\left\langle\frac{v}{\sigma(v)}\right\rangle,
\end{gathered}
$$

where $\langle g(v)\rangle=\frac{4 \pi}{n} \int_{0}^{\infty} g v^{2} \phi(\mathbf{y}, v, \tau) d v$ is the average over the velocity distribution. In the spatially homogeneous case eq. (17) coincides with the usual equation obtained in the two term approximation, whose stationary solution is

$$
\phi(v)=C \exp \left[-\int_{0}^{v} \frac{s^{3} d s}{\frac{k T}{m} s^{2}+\frac{1}{3}\left(\frac{e E^{*}}{m \rho \sigma}\right)^{2}}\right],
$$

which was given by Davydov in [Davydov 1935]. For $E^{*}=0 \phi(v)$ in $(21)$ is just $F(\mathbf{v} ; m)$ while for $T=0, \sigma=$ const it coincides with the Druyvesteyn distribution.

\section{Inequalities for moments at arbitrary $\epsilon$ and $\mathbf{T}=0$}

In previous works [Rokhlenko and Lebowitz 1997, Rokhlenko - submitted] we established bounds on the first few moments of the stationary distribution function using the approximate equation (3) with a velocity independent total cross section $\sigma_{0}$. Here we shall do the same for the $\operatorname{LBE}(2)$ when $T=0$. Introducing the dimensionless variables,

$$
x=\frac{\mathbf{v} \cdot \mathbf{E}}{v E}, \mathbf{s}=\mathbf{v} \epsilon^{1 / 4} \sqrt{\frac{m \rho \sigma_{0}}{e E}},
$$

and noting that for $T=0, F(\mathbf{V} ; M)=\delta(\mathbf{V})$, the stationary, spatially homogeneous, $f$ satisfies, in $d=3$, the equation,

$$
-\sqrt{\epsilon}\left(x \frac{\partial f}{\partial s}+\frac{1-x^{2}}{s} \frac{\partial f}{\partial x}\right)=
$$




$$
\frac{(1+\epsilon)^{2}}{2 \pi} \int f\left(\mathbf{s}^{\prime}\right) \delta\left[(1-\epsilon)\left(s^{\prime}\right)^{2}+2 \epsilon\left(\mathbf{s} \cdot \mathbf{s}^{\prime}\right)-(1+\epsilon) s^{2}\right] d \mathbf{s}^{\prime}-s f(\mathbf{s}) .
$$

Following [Lorentz 1916, Margenau and Hartman 1948, Rokhlenko 1991] we expand the distribution function in Legendre series

$$
f(s, x)=\sum_{l=0}^{\infty} f_{l}(s) P_{l}(x)
$$

substitute it into (22) and obtain a set of coupled equations for the $f_{l}$. Let us introduce the moments

$$
M_{l}(k)=\int_{0}^{\infty} s^{k} f_{l}(s) d s
$$

We can now use the same technique as in [Rokhlenko and Lebowitz 1997] to obtain bounds on the mean energy $W(\epsilon)$ and drift $u(\epsilon)$ which are defined in terms of $M_{0}(k)$. These inequalities are based on the fact that $\log M_{0}(k)$ is a convex function of $k$. This yield in the present case

$$
\frac{\sqrt{b}}{a} \leq \frac{2 \rho \sigma \sqrt{\epsilon}}{e E} W(\epsilon) \leq \frac{1}{\sqrt{a}}, \quad \frac{b^{1 / 4}}{a} \leq \frac{2 \epsilon^{3 / 4}}{\left|p_{0}(3, \epsilon)\right|} \sqrt{\frac{m \rho \sigma_{0}}{e E}} u(\epsilon) \leq \frac{1}{a^{3 / 4}}
$$

where

$$
a=\frac{p_{0}(4, \epsilon) p_{1}(2, \epsilon)}{3 \epsilon}, \quad b=\frac{p_{0}(7, \epsilon) p_{1}(5, \epsilon)}{12 \epsilon\left[1+p_{0}(3, \epsilon) / p_{2}(3, \epsilon)\right]}
$$

and

$$
p_{l}(k, \epsilon)=\frac{(1+\epsilon)^{2}}{2 \epsilon} \int_{|1-\epsilon / 1+\epsilon|}^{1} x^{k} P_{l}\left[\frac{(1+\epsilon) x^{2}+\epsilon-1}{2 \epsilon x}\right] d x-1 .
$$

When $\epsilon \rightarrow \infty$ the lower and upper bounds for $u$ and $W$ merge giving in the limit

$$
u=\sqrt{\frac{e E}{M \rho \sigma_{0}}}, \quad W=\frac{m u^{2}}{2} .
$$


When $\epsilon \rightarrow 0, a \rightarrow 1, \quad b \rightarrow 1 / 2$ and the inequalities (24) are then satisfied by the Druyvesteyn distribution. We believe that with greater effort it should be possible to obtain upper and lower bounds which when $\epsilon \rightarrow 0$ both converge to the values obtained from the Davydov distribution.

\section{Acknowledgments}

The research was supported by NSF Grant No. 95-23266 and AFOSR Grant No. 95-0159. 


\section{REFERENCES}

W.P.Allis, Handb.Phys. 21, 383 (1956)

N.Ben Abdollah and P.Degond, J.Math.Phys. 37, 3306 (1996)

N.Ben Abdollah, P.Degond, and S. Genies, J.Stat.Phys. 84, 205 (1996)

N.J.Carron, Phys.Rev.A 45, 2499-2511 (1991).

P.Davydov, Phys. Z. Sowjetunion 8, 59-70 (1935).

M.J.Druyvesteyn, Physica 10, 61 (1930)

M.J.Druyvesteyn and E.M.Penning, Rev.Mod.Phys. 12, 87 (1940)

R.Esposito, J.L.Lebowitz, and R.Marra, J.Stat.Phys. 78, 389 (1995)

R.N.Franklin, Plasma Phenomena in Gas Discharges (Clarendon, Oxford, 1976)

V.L.Ginzburg and A.V.Gurevich, Sov.Phys.-Usp. 3, 115 (1960)

V.E.Golant, A.P.Zhylinsky, and I.E.Sakharov, Fundamentals of Plasma Physics (Wiley, New York, 1980)

F.Golse and F.Poupaud, Asympt.Anal. 6, 135 (1992)

K.Koura, J.Phys.Soc.Japan 56, 429-432 (1987)

L.D.Landau and E.M.Lifshitz, Physical Kinetics (Pergamon Press, New York, 1993)

H.A.Lorentz, The Theory of Electrons (B.G.Taubner Leipzig, 1916)

H.Margenau and L.M.Hartman, Phys.Rev. 73, 309-315 (1948) 
A.Rokhlenko, Phys.Rev.A 43, 4438-4451 (1991)

A.Rokhlenko and J.L.Lebowitz, Phys.Rev.E, 56, 1012- 1018 (1997)

A.Rokhlenko, Phys.Rev.E (submitted and processed)

I.P.Shkarofsky, T.W.Johnston, and M.P.Bachynski, The Particle Kinetics of Plasma (AddisonWesley, Reading MA, 1966)

A.Sommerfeld, Thermodynamics and Statistical Mechanics (Acad. Press, New York, 1956)

H.Spohn, Large Scale Dynamics of Interacting Particles (Springen, 1995) 